\title{
Pr-magnetism in the quasi-skutterudite compound $\operatorname{PrFe}_{2} \mathrm{Al}_{8}$
}

\author{
Harikrishnan S. Nair, ${ }^{1,2, \text { * }}$ Michael O. Ogunbunmi, ${ }^{1}$ C. M. N. Kumar, ${ }^{3}$ D. \\ T. Adroja,${ }^{4}$ P. Manuel, ${ }^{4}$ D. Fortes, ${ }^{4}$ J. Taylor, ${ }^{4}$ and André M. Strydom ${ }^{1}$ \\ ${ }^{1}$ Highly Correlated Matter Research Group, Physics Department, \\ P. O. Box 524, University of Johannesburg, Auckland Park 2006, South Africa \\ ${ }^{2}$ Department of Physics, Colorado State University, 200 W. Lake St., Fort Collins, CO 80523-1875, USA \\ ${ }^{3}$ Laboratoire National des Champs Magnétiques Intenses, \\ CNRS-INSA-UJF-UPS, 143 Avenue de Rangueil, F-31400 Toulouse, France \\ ${ }^{4}$ ISIS Facility, STFC, Rutherford Appleton Laboratory, \\ Chilton, Didcot, Oxfordshire OX11 OQX, United Kingdom
}

\begin{abstract}
The intermetallic compound $\mathrm{PrFe}_{2} \mathrm{Al}_{8}$ that possesses a three-dimensional network structure of $\mathrm{Al}$ polyhedra centered at the transition metal element Fe and the rare earth Pr is investigated through neutron powder diffraction and inelastic neutron scattering in order to elucidate the magnetic ground state of $\mathrm{Pr}$ and $\mathrm{Fe}$ and the crystal field effects of Pr. Our neutron diffraction study confirms long-range magnetic order of Pr below $T_{N}=4.5 \mathrm{~K}$ in this compound. Subsequent magnetic structure estimation reveals a magnetic propagation vector $\mathbf{k}=\left(\frac{1}{2} \mathbf{0} \frac{1}{2}\right)$ with a magnetic moment value of $2.5 \mu_{\mathrm{B}} / \mathrm{Pr}$ along the orthorhombic $c$-axis and evidence the lack of ordering in the Fe sublattice. The inelastic neutron scattering study reveals one crystalline electric field excitation near $19 \mathrm{meV}$ at $5 \mathrm{~K}$ in $\mathrm{PrFe}_{2} \mathrm{Al}_{8}$. The energy-integrated intensity of the $19 \mathrm{meV}$ excitation as a function of $|Q|\left(\AA^{-1}\right)$ follows the square of the magnetic form factor of $\operatorname{Pr}^{3+}$ thereby confirming that the inelastic excitation belongs to the Pr sublattice. The second sum rule applied to the dynamic structure factor indicates only $1.6(2) \mu_{\mathrm{B}}$ evolving at the $19 \mathrm{meV}$ peak compared to the $3.58 \mu_{\mathrm{B}}$ for free $\mathrm{Pr}^{3+}$, indicating that the crystal field ground state is magnetic and the missing moment is associated with the resolution limited quasi-elastic line. The magnetic order occurring in $\mathrm{Pr}$ in $\mathrm{PrFe}_{2} \mathrm{Al}_{8}$ is counter-intuitive to the symmetry-allowed crystal field level scheme, hence, is suggestive of exchange-mediated mechanisms of ordering stemming from the magnetic ground state of the crystal field levels.

PACS numbers:
\end{abstract}

\section{INTRODUCTION}

Intermetallic compounds possessing a cage-like crystal structure comprising of polyhedra that contain rare earth or transition metal atoms in their voids have been interesting compounds from the perspective of thermoelectric properties, however, the magnetism of rare earth that is placed in the cages is a relatively less-explored topic. The filledskutterudites $R T_{4} X_{12}$ where $R=$ rare earth, $T=$ transition metal and $X=$ pnictogen, is a well-explored class of compounds [1, 2] with the potential of making thermoelectric applications [3]. The original binary skutterudite formula can be written as $T X_{3}$ which, with the existence of voids in the original $\operatorname{Im} 3$ structure, may adopt a general formula $R_{2} M_{8} X_{24}$ or $R M_{4} X_{12}$. The cubic $R T_{2} \mathrm{Al}_{20}$, known as the Frank Kasper compounds, provide another example of the caged-structure compounds similar to the filled-skutterudites, and have a large coordination number of atoms constructing the cage around the $R$ atom[4]. They crystallize in the space group $F d \overline{3} m$ (\#227) with the $R$ ion surrounded by neighboring 16 atoms and the $R$ site is of a cubic point symmetry. A related cagedstructure compound is the $R T_{2} \mathrm{Al}_{10}$ which has orthorhombic crystal structure with the space group $C m c m$ (\#63) [5, 6]. In this caged system the $R$ atoms are 20-fold coordinated and are located inside $T-\mathrm{Al}$ cages. It is interesting to look at the magnetism of these caged-network compounds with Pr occupying

\footnotetext{
*Electronic address: h.nair.kris@gmail.com, hsnair@colostate.edu
}

the void inside the cage. For example, the filled-skutterudite $\mathrm{PrRu}_{4} \mathrm{Sb}_{12}$ shows a Van Vleck paramagnetic behaviour for most of the temperature range before entering a superconducting phase below $1.04 \mathrm{~K}[7,8]$. In the case of $\operatorname{PrTi}_{2} \mathrm{Al}_{20}$, a non-magnetic order parameter was identified below the magnetic transition at $2 \mathrm{~K}[9]$. This was correlated with the ferroquadrupole ordering scenario. In the case of $\operatorname{PrRu}_{2} \mathrm{Al}_{10}$, which displayed metallic behaviour, no magnetic phase transition was recorded down to low temperature[10]. These studies focusing on the rare earth magnetism in the caged-network points towards the interesting magnetic ordering or the lack of it, that may have a strong influence from the ligand field around the rare earth. The crystal field ground state of the $4 f$ ion in the cages play an important role in the magnetic ordering phenomena in these compounds. While many studies focused on the magnetism of the $4 f$ element in the cages, it must be noted that the magnetism of the $3 d$ element and the correlation between the two is an emergent theme in similar compounds. For example, ab-initio calculations in the $\mathrm{Ce}_{3} T_{4} X_{13}$ compounds have shown indication of correlation between the $3 d$ and the $4 f$ wave functions[11]. Unexpected localization of Fe $3 d$ moments were experimentally observed in another caged compound $\mathrm{Yb}_{2} \mathrm{Fe}_{2} \mathrm{Al}_{10}$ where it was observed that the long-range ordering of the $3 d$ moments were avoided down to $0.4 \mathrm{~K}[12]$. These studies point to case of caged intermetallics where the rare earth and transition metal display cooperative phenomena in concert or as separate. With this background, we take a look at a rather less-studied caged-network intermetallic compound, $\mathrm{PrFe}_{2} \mathrm{Al}_{8}$ that belongs to the family of $R T_{2} X_{8}(X=\mathrm{Al}, \mathrm{Ga})$. 


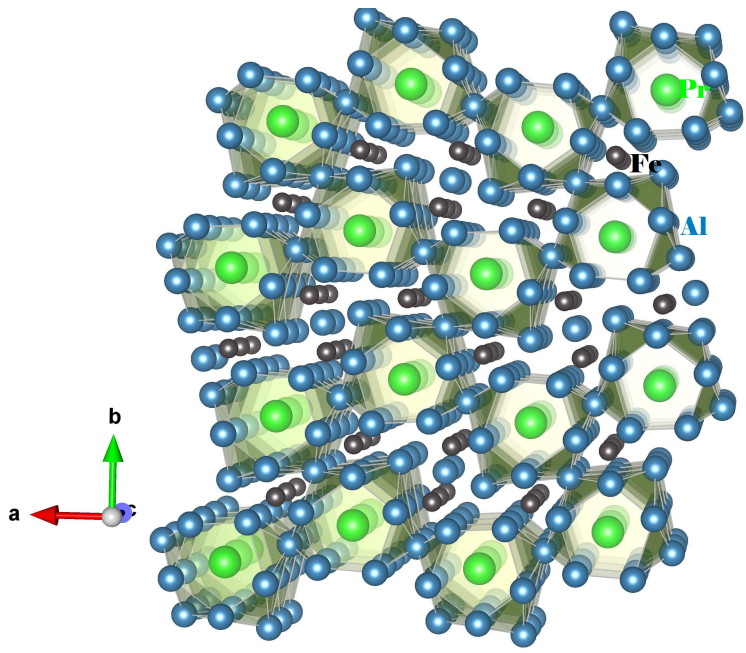

FIG. 1: (color online) A schematic representation of the cage-like network structure of the quasi-skutterudite $\mathrm{PrFe}_{2} \mathrm{Al}_{8}$. A projection of the cages on the $a b$ plane is shown here. Note that the representation is not a unit cell. The green spheres represent Pr while the dark gray are $\mathrm{Fe}$ and the turquoise are $\mathrm{Al}$.

The $R T_{2} X_{8}$ compounds crystallize in cubic Pbam space group. Figure 1 shows the projection of the crystal structure on $a b$-plane displaying the network of $\mathrm{Al}$ and the chain-like formation of $\mathrm{Pr}$ and Fe along the $c$-axis. These structural peculiarities certainly have a bearing on the magnetism of this class of compounds, notably, a strong influence from crystal field effects. Depending on whether the crystal field levels are comparable in energy scales with other interactions (especially, magnetic exchange), a non-magnetic singlet or doublet crystal field ground state can result. To cite an exam-

TABLE I: The lattice parameters of $\mathrm{PrFe}_{2} \mathrm{Al}_{8}$ obtained from the refinement of neutron diffraction data at 300,150 and $25 \mathrm{~K}$ are provided in the top section of the table. The refined atomic coordinates of $\mathrm{PrFe}_{2} \mathrm{Al}_{8}$ at $300 \mathrm{~K}$ are given in the bottom section. These values are obtained from the HRPD-data.

\begin{tabular}{rrrrrr}
\hline \hline & \multicolumn{2}{c}{$300 \mathrm{~K}$} & $150 \mathrm{~K}$ & \multicolumn{2}{l}{$25 \mathrm{~K}$} \\
\hline \hline$a(\AA)$ & $12.5101(3)$ & $12.4871(5)$ & $12.4799(4)$ \\
$b(\AA)$ & $14.4383(3)$ & $14.4168(6)$ & $14.4081(8)$ \\
$c(\AA)$ & $4.0411(1)$ & $4.0332(8)$ & $4.0297(2)$ \\
\hline \multicolumn{5}{c}{$\mathrm{PrFe}_{2} \mathrm{Al}_{8}}$, & $P b a m, 300 \mathrm{~K}$ \\
\hline Atom & site & $x$ & $y$ & $z$ & $U_{\text {iso }}$ \\
\hline \hline $\mathrm{Pr}$ & $4 g$ & 0.34430 & 0.31793 & 0.0 & 0.0029 \\
$\mathrm{Fe}(1)$ & $4 g$ & 0.15095 & 0.09235 & 0.0 & 0.0018 \\
$\mathrm{Fe}(2)$ & $4 g$ & 0.03619 & 0.40434 & 0.0 & 0.0021 \\
$\mathrm{Al}(1)$ & $2 d$ & 0.0000 & 0.5000 & 0.5 & 0.0033 \\
$\mathrm{Al}(2)$ & $2 a$ & 0.0000 & 0.0000 & 0.0 & 0.0053 \\
$\mathrm{Al}(3)$ & $4 h$ & 0.02968 & 0.13355 & 0.5 & 0.0053 \\
$\mathrm{Al}(4)$ & $4 h$ & 0.16160 & 0.3761 & 0.5 & 0.0031 \\
$\mathrm{Al}(5)$ & $4 h$ & 0.23677 & 0.17102 & 0.5 & 0.0035 \\
$\mathrm{Al}(6)$ & $4 h$ & 0.33260 & 0.49029 & 0.5 & 0.0034 \\
$\mathrm{Al}(7)$ & $4 h$ & 0.45987 & 0.18346 & 0.5 & 0.0028 \\
$\mathrm{Al}(8)$ & $4 g$ & 0.33473 & 0.03719 & 0.0 & 0.0041 \\
$\mathrm{Al}(9)$ & $4 g$ & 0.09153 & 0.25152 & 0.0 & 0.0043 \\
\hline \hline
\end{tabular}

TABLE II: The Pr-Pr and the Fe-Fe nearest-neighbour distances and angles of $\mathrm{PrFe}_{2} \mathrm{Al}_{8}$ at $300 \mathrm{~K}, 150 \mathrm{~K}, 25 \mathrm{~K}$ and $1.6 \mathrm{~K}$. The distances along $a, b$ and $c$ directions are shown. The angle shown is that which is formed between any three nearest-neighbour Pr atoms.

\begin{tabular}{lcccc}
\hline & $300 \mathrm{~K}$ & $150 \mathrm{~K}$ & $25 \mathrm{~K}$ & $1.6 \mathrm{~K}$ \\
\hline \hline $\operatorname{Pr}-\operatorname{Pr}(\| c)$ & $4.0411(9) \AA$ & $4.0334(6) \AA$ & $4.0297(2) \AA$ & $4.0297(2) \AA$ \\
$\operatorname{Pr}-\operatorname{Pr}(\| a)$ & $6.572(9) \AA$ & $6.562(11) \AA$ & $6.559(11) \AA$ & $6.554(13) \AA$ \\
$\operatorname{Pr}-\operatorname{Pr}(\| b)$ & $7.563(7) \AA$ & $7.554(7) \AA$ & $7.551(9) \AA$ & $7.560(9) \AA$ \\
\hline $\mathrm{Fe}-\mathrm{Fe}(\| c)$ & $4.0411(2) \AA$ & $4.0335(6) \AA$ & $4.0297(6) \AA$ & $4.0297(8) \AA$ \\
$\mathrm{Fe}-\mathrm{Fe}(\| a)$ & $4.818(14) \AA$ & $4.815(4) \AA$ & $4.816(5) \AA$ & $4.817(6) \AA$ \\
$\mathrm{Fe}-\mathrm{Fe}(\| b)$ & $2.754(4) \AA$ & $2.748(5) \AA$ & $2.757(5) \AA$ & $2.762(7) \AA$ \\
\hline$\angle \operatorname{Pr}(\| a)$ & $144.23(8)^{\circ}$ & $144.12(7)^{\circ}$ & $144.12(7)^{\circ}$ & $144.36(9)^{\circ}$ \\
$\angle \operatorname{Pr}(\| b)$ & $145.32(9)^{\circ}$ & $145.19(11)^{\circ}$ & $145.15(10)^{\circ}$ & $145.68(11)^{\circ}$ \\
\hline
\end{tabular}

ple, the point charge model calculations that take in to account the local symmetry for the Pr site in PrSi predicts a splitting of $J=4 \mathrm{Pr}^{3+}$ ground state in to 9 singlets which renders spontaneous magnetic order impossible. However, an anomalous ferromagnetic ground state (with an ordering temperature of $\approx 55 \mathrm{~K}$ ) was experimentally unraveled in $\operatorname{PrSi}[13]$. Similarly, the point symmetry of the Pr-site in $\mathrm{PrFe}_{2} \mathrm{Al}_{8}$ is $m$ $\left(C_{s}\right)$ which predicts 9 distinct singlets from a 9 -fold degenerate state which consequentially presupposes a non-magnetic ground state. Our previous studies on $\mathrm{PrFe}_{2} \mathrm{Al}_{8}$ had revealed long-range magnetic order through macroscopic magnetization measurements which was assumed to have originated from the Pr magnetic sublattice[14]. The Fe sublattice was deduced to be non-magnetic or as having only short-range spin correlations. In the present work, we investigate the magnetic ground state of $\mathrm{PrFe}_{2} \mathrm{Al}_{8}$ through neutron diffraction experiments and confirm that only Pr orders magnetically longrange while Fe is not. In addition, our study sheds light on the magnetic excitations and crystal field effects through inelastic neutron scattering.

\section{EXPERIMENTAL DETAILS}

About $10 \mathrm{~g}$ of polycrystalline powder of $\mathrm{PrFe}_{2} \mathrm{Al}_{8}$ and $\mathrm{LaFe}_{2} \mathrm{Al}_{8}$ were prepared following the procedure outlined in Ref.[14]. The powder samples were initially characterized using laboratory $\mathrm{x}$-rays to check for purity of the chemical phase and using magnetometry to identify the magnetic phase transition. These preliminary checks confirmed the findings of Ref.[14]. Time-of-flight (TOF) neutron powder diffraction experiments were carried out at the instrument HRPD at ISIS facility of the Rutherford Appleton Laboratory, UK mainly to characterize the temperature evolution of the crystal structure. Diffraction patterns covering a $d$-spacing range $0.75-4 \AA$ were obtained at 19 temperature points in the interval of $300 \mathrm{~K}$ to $1.6 \mathrm{~K}$. Additionally, experiments were also performed using the TOF diffractometer WISH on TS2 at ISIS facility especially covering the higher $d$-spacing range of $1.2-11 \AA$ in order to unambiguously determine the magnetic structure of $\mathrm{PrFe}_{2} \mathrm{Al}_{8}$. Inelastic neutron scattering experiments were performed on $10 \mathrm{~g}$ of $\mathrm{PrFe}_{2} \mathrm{Al}_{8}$ powder sample using the TOF chopper spectrometer MARI at ISIS facility. $\mathrm{PrFe}_{2} \mathrm{Al}_{8}$ pow- 
der from the same batch was used in all the three experiments. Incident neutron energies $E_{i}=8 \mathrm{meV}, 50 \mathrm{meV}$ and $150 \mathrm{meV}$ were selected for the experiment. The chopper frequencies were set at $150 \mathrm{~Hz}, 250 \mathrm{~Hz}$, and $400 \mathrm{~Hz}$, respectively. Data were collected mainly at $5 \mathrm{~K}, 50 \mathrm{~K}, 200 \mathrm{~K}$ and at $300 \mathrm{~K}$. As a non-magnetic reference, the inelastic response of $\mathrm{LaFe}_{2} \mathrm{Al}_{8}$ was also recorded to serve as a phonon estimate for $\mathrm{PrFe}_{2} \mathrm{Al}_{8}$. The inelastic neutron data was analyzed using the software DAVE[15]. Rietveld refinement[16] of the diffraction data were carried out using FULLPROF software [17]. The software SARA $h[18]$ was used for the representation analysis of magnetic structure from diffraction data.

\section{RESULTS}

\section{A. Neutron Diffraction}

A $2 \mathrm{D}$ contour plot of the diffracted intensity from $\mathrm{PrFe}_{2} \mathrm{Al}_{8}$ obtained from the diffraction data of bank-3 of HRPD, is shown in figure 2 as a function of temperature and $d$-spacing. Diffracted intensity due to Bragg scattering from the nuclear structure is present at $d \approx 4 \AA, 5.5 \AA, 6.2 \AA, 7.2 \AA$ and $9.5 \AA$. It is observed that additional magnetic intensity develops only below $4 \mathrm{~K}$, at the $d$-spacing $5.3 \AA$, which is indicated in figure 2 by a horizontal white dashed-line. Note that the temperature-axis is plotted in log-scale for clarity. According to our previous investigation on $\mathrm{PrFe}_{2} \mathrm{Al}_{8}$ [14], longrange magnetic order sets in only below $T_{N}^{P r} \approx 4 \mathrm{~K}$. The experimental evidence in figure 2 supports this assignment of magnetic phase transition temperature $T_{N}^{P r} \approx 4 \mathrm{~K}$ for Pr. In our previous work signatures akin to short-range spin fluctuations in the Fe lattice were observed in magnetic susceptibility at $T_{\text {anom }} \approx 34 \mathrm{~K}$. No magnetic scattered intensity is developed at around $34 \mathrm{~K}$ in the present diffraction data thereby ruling out the presence of magnetic ordering on the Fe lattice in this compound. More concretely, in a later subsection of the present paper, it is shown through inelastic neutron data that the energy-integrated intensity as a function of $|Q|$ follows the magnetic form factor of $\mathrm{Pr}^{3+}$ there by confirming that only $\mathrm{Pr}$ orders magnetically in $\mathrm{PrFe}_{2} \mathrm{Al}_{8}$.

The neutron powder diffraction intensity pattern of $\mathrm{PrFe}_{2} \mathrm{Al}_{8}$ at $25 \mathrm{~K}$ obtained from HRPD, is plotted in figure 3 (a) (black circles) as a function of $d$-spacing. The red solid line in the figure is the result of a Rietveld fit to the data using Pbam space group. From the magnetization measurements we know that at $25 \mathrm{~K} \mathrm{PrFe}_{2} \mathrm{Al}_{8}$ is not magnetically ordered and hence the diffraction data was modeled using only the nuclear structure in the space group model Pbam as has been used in our previous work [14]. The diffracted intensity at $300 \mathrm{~K}$ (not shown) is similar to that at $25 \mathrm{~K}$ as presented in figure 3 (a) and no signature of any structural phase transition is observed in this temperature interval of $300-25 \mathrm{~K}$. The lattice parameters $a, b$ and $c$ show a gradual decrease with temperature as can be seen from the values at $300 \mathrm{~K}, 150 \mathrm{~K}$ and $25 \mathrm{~K}$ collected in Table (I). Also presented in Table (I) are the atomic parameters of $\mathrm{PrFe}_{2} \mathrm{Al}_{8}$ at $300 \mathrm{~K}$. The crystal structure of $\mathrm{PrFe}_{2} \mathrm{Al}_{8}$ in $\mathrm{Pbam}$ space group has $\mathrm{Pr}$ occupying

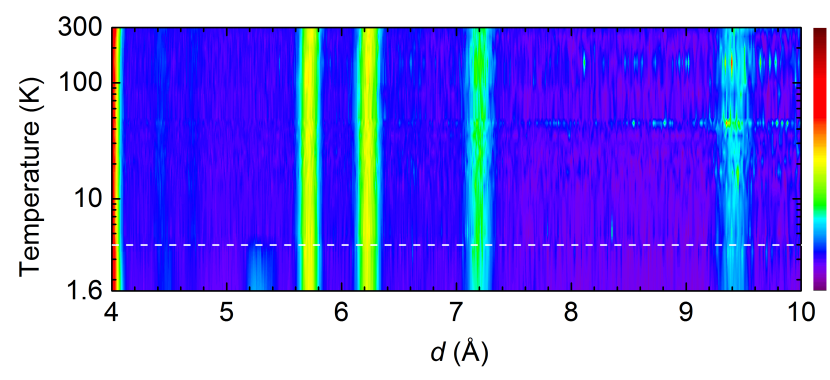

FIG. 2: (color online) A colour plot of diffracted intensity from $\mathrm{PrFe}_{2} \mathrm{Al}_{8}$ measured at HRPD, plotted as a function of temperature versus $d$-spacing. The temperature axis is shown in log scale. A horizontal dashed line marks $T_{N}^{\mathrm{Pr}} \approx 4 \mathrm{~K}$ where the magnetic long-range order of Pr begins as evidenced by the development of intensity at $d \approx 5.3 \AA$.

a single Wyckoff position $4 g[19]$. The crystal structure is a close-packed arrangement of three dimensional Al polyhedra which are packed face-sharing to form chains of $\mathrm{Pr}$ and $\mathrm{Fe}$ along the $c$-axis separated from each other by the $\mathrm{Fe}$-Al network (see figure 1). The shortest $\mathrm{Pr}-\mathrm{Pr}$ and Fe-Fe distances are parallel to the $c$-axis. As a function of temperature, the Pr-Pr and the Fe-Fe nearest-neighbour distances undergo only slight variation. A slight decrease in the nearest-neighbour distances is observed in all the directions $a, b$ and $c$. The bond angles between the nearest Pr atoms also do not vary significantly down till $1.6 \mathrm{~K}$. Along $a$ and $b$ directions, they remain close to the values $144^{\circ}$ and $145^{\circ}$ respectively. The bond parameters as a function of temperature are collected in Table (II).

One of the important questions about the magnetism of $\mathrm{PrFe}_{2} \mathrm{Al}_{8}$ is whether $\mathrm{Pr}$ and $\mathrm{Fe}$ in this compound order in a magnetically long-range fashion, either individually or jointly, or not. In our previous study[14], magnetic anomalies at $T_{\text {anom }} \approx 34 \mathrm{~K}$ and $T_{N}^{\mathrm{Pr}} \approx 4 \mathrm{~K}$ were observed in the magnetization measurements. The anomaly at $T_{\text {anom }}$ was attributed to short-range order in the Fe lattice or due to minute amount of magnetic impurity; whereas $T_{N}^{\mathrm{Pr}}$ was attributed to longrange order in the Pr magnetic lattice. Hence, one of the major aims of our neutron diffraction experiments was to determine the microscopic nature of the magnetic phase transitions pertaining to $\mathrm{Fe}$ or $\mathrm{Pr}$ in $\mathrm{PrFe}_{2} \mathrm{Al}_{8}$. Since, through the low angle bank of HRPD, the development of long-range magnetic order in the Pr sublattice was observed to occur below $4 \mathrm{~K}$, we performed a detailed low temperature neutron diffraction measurements using the WISH diffractometer in the temperature range $2 \mathrm{~K}$ to $50 \mathrm{~K}$ covering a larger $d$-spacing up to $11 \AA$. The diffracted intensity at $50 \mathrm{~K}$ was identical to that of $20 \mathrm{~K}$. No additional magnetic Bragg peaks or short range magnetic diffuse scattering were observed at $20 \mathrm{~K}$. Also, there was no enhancement of diffracted intensity at low temperatures suggesting ferromagnetic contributions at nuclear Bragg positions. These results confirm that the $34 \mathrm{~K}$ transition observed previously [14] in the magnetic susceptibility is not from the bulk of the sample.

The neutron diffraction patterns at $20 \mathrm{~K}$ and $2 \mathrm{~K}$ obtained from WISH along with the Rietveld refinement results are presented in figure 3 (b), (c) and (d). It can be readily ob- 

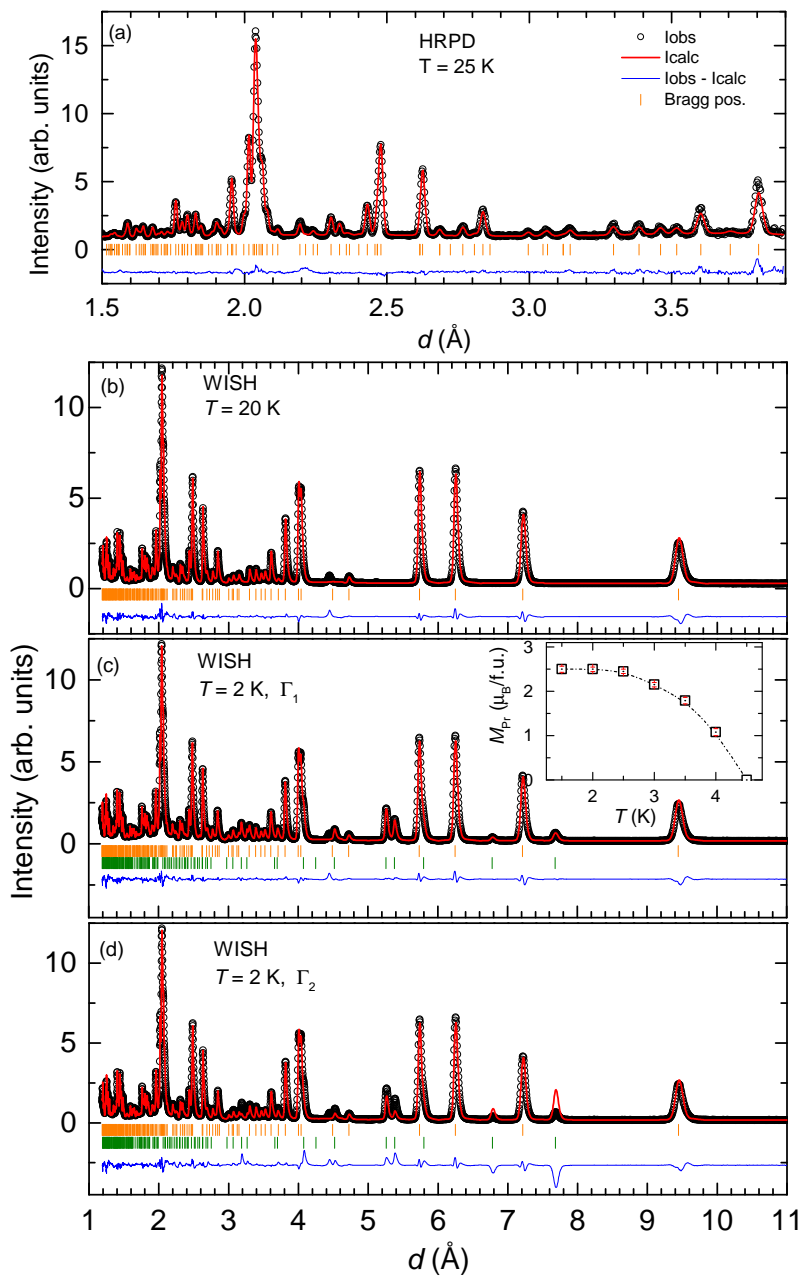

FIG. 3: (color online) (a) Neutron powder diffraction intensity of $\mathrm{PrFe}_{2} \mathrm{Al}_{8}$ at $25 \mathrm{~K}$ (circles) obtained from the time-of-flight diffractometer HRPD at ISIS facility, UK. Low temperature neutron diffraction pattern of $\mathrm{PrFe}_{2} \mathrm{Al}_{8}$ at (b) $20 \mathrm{~K}$ and at (c) $2 \mathrm{~K}$ obtained from the diffractometer WISH at ISIS facility, UK. A larger coverage of $d$-spacing helps in elucidating the magnetic structure of $\operatorname{Pr}$ in this compound. In panel (b), the lower set of vertical tick marks correspond to the Bragg peaks of the magnetic structure according to $\Gamma_{1}$ representation. The inset to (c) presents the temperature dependence of refined value of magnetic moment of $\mathrm{Pr}^{3+}$ in $\mathrm{PrFe}_{2} \mathrm{Al}_{8}$ showing a phase transition at $T_{N}^{\mathrm{Pr}}=4.5 \mathrm{~K}$. (d) The $2 \mathrm{~K}$ WISH data refined using the incorrect model $\Gamma_{2}$ does not account for the intensities correctly.

served that additional scattered intensity has developed at $2 \mathrm{~K}$ as compared to the intensity profiles at high temperatures. Especially, new peaks at $d \approx 5.25 \AA, 5.37 \AA, 6.78 \AA$ and $7.68 \AA$ emerge. The nuclear structure below $T_{N}^{P r} \approx 4 \mathrm{~K}$ conforms to Pbam, which is the crystal structure at $300 \mathrm{~K}$. Hence, any structural phase transformation in the temperature range 300 $4 \mathrm{~K}$ is ruled out. In order to ascertain the ordering is from Pr or Fe or from both in $\mathrm{PrFe}_{2} \mathrm{Al}_{8}$ first, a determination of the magnetic propagation vector was undertaken. Using the $k$-search utility in the FULLPROF suite of programs, the most probable propagation vector was determined as $\mathrm{k}\left(\frac{1}{2} \mathbf{0} \frac{1}{2}\right)$ to be used for the representation analysis of magnetic structure. Ac-
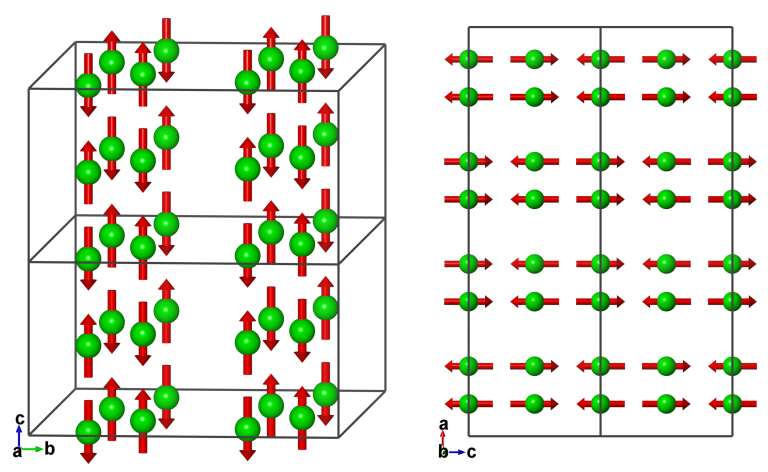

FIG. 4: (color online) The magnetic structure of $\mathrm{PrFe}_{2} \mathrm{Al}_{8}$ according to the $\Gamma_{1}$ representation. The $\operatorname{Pr}^{3+}$ moments are shown as red arrows ordering antiferromagnetically. Two different views of a $1 \times 1 \times 2$ unit cell projected on to the plane are presented.

cording to the symmetry analysis for the orthorhombic Pbam space group, two magnetic representations $\Gamma_{1}$ and $\Gamma_{2}$ - both antiferromagnetic - were found as acceptable solutions for the magnetic structure of $\operatorname{Pr}^{3+}$ in $\mathrm{PrFe}_{2} \mathrm{Al}_{8}$. Hence, Rietveld refinement of diffracted intensity at $2 \mathrm{~K}$ was performed by assuming the nuclear space group as Pbam and the magnetic representation as $\Gamma_{1}$ or $\Gamma_{2}$. The magnetic $R$-factor for the refinements with $\Gamma_{1}$ or $\Gamma_{2}$ were 3.4 and 80 respectively, indicating that $\Gamma_{1}$ is the best fit. In this representation, Pr moments order antiferromagnetically with moment direction along the orthorhombic $c$-axis. In the panel (d) of Figure 3, the Rietveld refinement of $2 \mathrm{~K}$ data of $\mathrm{PrFe}_{2} \mathrm{Al}_{8}$ is shown, with the fits using $\Gamma_{2}$ representation (lower tick marks). Noticeably, the intensities are not correctly accounted for in this model (near $d \approx 5.5 \AA$ And $7.7 \AA$ ). A refinement model that allows for long-range magnetic order in both $\mathrm{Pr}$ and $\mathrm{Fe}$ lattices did not lead to an improved fit and hence was discarded. The experimental data did not fit to a model that considers only the $\mathrm{Fe}$ moment ordering, either. Moreover, from our previous experimental study[14] it was understood that the magnetic anomaly tentatively attributed to $\mathrm{Fe}$ at $T_{\text {anom }}$ was very broad suggesting short-range correlations to be present rather than a conventional paramagnet-to-antiferromagnet phase transition. A value of $2.5 \mu_{\mathrm{B}} / \mathrm{Pr}$ is obtained at $2 \mathrm{~K}$ for the neutronderived ordered moment of Pr. A gradual decrease of the ordered magnetic moment was observed until $T_{N}^{P r}=4.5 \mathrm{~K}$. The temperature-evolution of the ordered moment of $\operatorname{Pr}, M_{\mathrm{Pr}}$, is presented in the inset of figure 3 (c). The dashed line in the inset serves as a guide to eye. The antiferromagnetic $\Gamma_{1}$ magnetic structure adopted by $\mathrm{Pr}^{3+}$ moments in $\mathrm{PrFe}_{2} \mathrm{Al}_{8}$ is pictured in figure 4

\section{B. Inelastic Neutron Scattering}

Once we determined the magnetic structure of $\mathrm{PrFe}_{2} \mathrm{Al}_{8}$ we now proceed to study the magnetic excitations and the crystal field effects using inelastic neutron scattering methods. The inelastic neutron scattering intensity of $\mathrm{PrFe}_{2} \mathrm{Al}_{8}$ at 

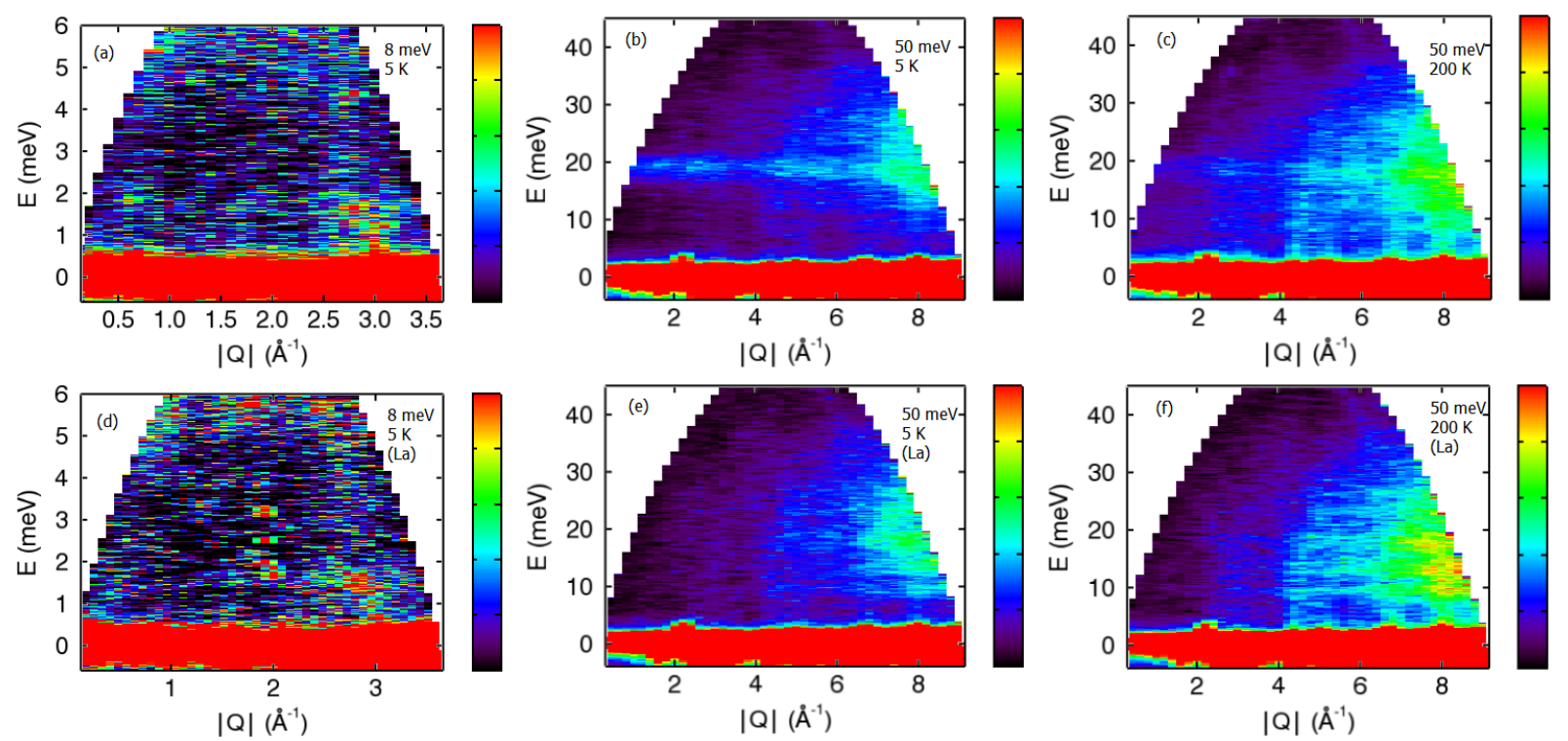

FIG. 5: (color online) The color-coded plot of the inelastic neutron scattering intensity of $\mathrm{PrFe}_{2} \mathrm{Al}_{8}$ measured at MARI plotted as energy transfer (E) versus momentum transfer ( $|\mathrm{Q}|$ ) at (a) $5 \mathrm{~K}, E_{i}=8 \mathrm{meV}$ (b) $5 \mathrm{~K}, E_{i}=50 \mathrm{meV}$ and (c) $200 \mathrm{~K}, E_{i}=50 \mathrm{meV}$; all three for $\mathrm{PrFe}_{2} \mathrm{Al}_{8}$ (d) $5 \mathrm{~K}, E_{i}=8 \mathrm{meV}$ (e) $50 \mathrm{~K}, E_{i}=50 \mathrm{meV}$ and (f) $200 \mathrm{~K}, E_{i}=50 \mathrm{meV}$ for $\mathrm{LaFe}_{2} \mathrm{Al}_{8}$. An inelastic excitation is observed at $E \approx 19 \mathrm{meV}$ for $\mathrm{PrFe}_{2} \mathrm{Al}_{8}$ (in (b)) which is attributed to crystal field effects. The intensity axes were normalized to a scale of $50 \mathrm{in}$ the units of mbr/sr/meV/fu.

different incident neutron energies and temperatures are presented in figure 5 (a) through (f) where color maps of energy transfer (in meV) versus momentum transfer $\left(|Q|\right.$ in $\AA^{-1}$ ) are shown. Specifically, (a) $E_{i}=8 \mathrm{meV}, T=5 \mathrm{~K}$, (b) $E_{i}=$ $50 \mathrm{meV}, T=5 \mathrm{~K}$ and (c) $E_{i}=50 \mathrm{meV}, T=200 \mathrm{~K}$ for $\mathrm{PrFe}_{2} \mathrm{Al}_{8}$ are shown. The inelastic intensity of the non-magnetic reference compound $\mathrm{LaFe}_{2} \mathrm{Al}_{8}$ are shown in (d) $E_{i}=8 \mathrm{meV}, T=$ $5 \mathrm{~K}$, (e) $E_{i}=50 \mathrm{meV}, T=5 \mathrm{~K}$ and (f) $E_{i}=50 \mathrm{meV}, T=$ $200 \mathrm{~K}$. The intensity pattern at $5 \mathrm{~K}$ and at $200 \mathrm{~K}$ are essentially identical, suggesting that there is no magnetic low lying excitations in the energy range approximatley up to $10 \mathrm{meV}$. The absence of any excited state transition at $200 \mathrm{~K}$ suggests that there are no CEF levels below $15 \mathrm{meV}$, which have nonzero matrix elements. At low energy transfer, for example with $E_{i}=8 \mathrm{meV}$ (shown in (a)), no magnetic inelastic excitations are observed in $\mathrm{PrFe}_{2} \mathrm{Al}_{8}$. This result indicates that the quasi-elastic linewidth is very small and not able to detect within the resolution of MARI ( $\Delta E=0.19 \mathrm{meV}$ for $E_{i}$ $=8 \mathrm{meV}, 150 \mathrm{~Hz}$ ). In figure 5, only one inelastic excitation is seen present at all the temperatures, viz., at $19 \mathrm{meV}$ which arises from the crystal field excitation of the $\mathrm{Pr}^{3+}$ crystal field levels, as can be seen from the panel (b) with incoming energy, $E_{i}=50 \mathrm{meV}$ at $5 \mathrm{~K}$. The inelastic spectra shown in figure 5 also suggest the absence of magnetic ordering down to $5 \mathrm{~K}$, as one would expect spin wave excitations in an ordered state. Further the $|Q|$-dependent cuts (not shown) obtained by binning the energy from $-2 \mathrm{meV}$ to $2 \mathrm{meV}$ (from (a) and (b)) did not reveal any magnetic Bragg peaks at $5 \mathrm{~K}$. From panels (d, e) that represent the inelastic scattered intensity of $\mathrm{LaFe}_{2} \mathrm{Al}_{8}$ at $5 \mathrm{~K}$, it is clear that no magnetic intensity is present in the non-magnetic analogue compound, which indicates that the $\mathrm{Fe}$ atoms do not have ordered magnetic moments in $\mathrm{LaFe}_{2} \mathrm{Al}_{8}$ or $\operatorname{PrFe}_{2} \mathrm{Al}_{8}$. An $E$ versus $|Q|$ plot of $\mathrm{PrFe}_{2} \mathrm{Al}_{8}$ obtained using
$E_{i}=150 \mathrm{meV}$ (not shown here) also did not provide any evidence of other CEF excitations between $20 \mathrm{meV}$ and $145 \mathrm{meV}$. The high intensity observed in (d) towards the high- $Q$ originates from the phonon contribution. The inelastic intensity of $\mathrm{PrFe}_{2} \mathrm{Al}_{8}$ at $200 \mathrm{~K}$ (in (c)) is compared with that of $\mathrm{LaFe}_{2} \mathrm{Al}_{8}$ in the panels (e) and (f), which further confirmed that high- $|Q|$ intensity is mainly dominated by the phonon scattering as its intensity shows an increase with increasing temperature.

The $|Q|$-integrated (0 to $3 \AA^{-1}$ ) dynamic structure factor, $S(Q, \omega)$, for $\mathrm{PrFe}_{2} \mathrm{Al}_{8}$ as a function of energy transfer at $E_{i}=$ $50 \mathrm{meV}$ is presented in figure 6 (a) for $5 \mathrm{~K}, 50 \mathrm{~K}$ and $200 \mathrm{~K}$. The location in the graph where energy transfer equals zero corresponds to the elastic line. The inelastic signal arising from the crystal field effect is observed at $19 \mathrm{meV}$ which diminishes in intensity as temperature is raised. The $S(Q, \omega)$ dependence on energy transfer with $E_{i}=8 \mathrm{meV}$ is shown in the inset (a1), which shows the absence of any excitations in the energy range accessed. The dynamic structure factor for inelastic neutron scattering of unpolarized neutrons is given by the equation[20],

$$
S(Q, \omega)=\frac{\hbar \omega}{1-\exp \left(-\hbar \omega / k_{\mathrm{B}} T\right)} F^{2}(Q) \chi_{0} P(Q, \hbar \omega)
$$

where $F(Q)$ is the magnetic form factor of the magnetic ion, $\chi_{0}$ is the static magnetic susceptibility and $P(Q, \hbar \omega)$ is a spectral function. The first term on the right-hand-side is the Bose factor for the thermal occupation of levels. Curve fits to the $19 \mathrm{meV}$ excitation according to Equation (1) is shown in Figure 6(b) for $5 \mathrm{~K}, 50 \mathrm{~K}$ and $200 \mathrm{~K}$. The FWHM of the peaks as well as the area under the peak which corresponds to the local susceptibility were extracted from these fits and presented respectively in panels (c) and (d) of Figure 6 A gradual broadening of the peaks with increasing temperature is observed, 

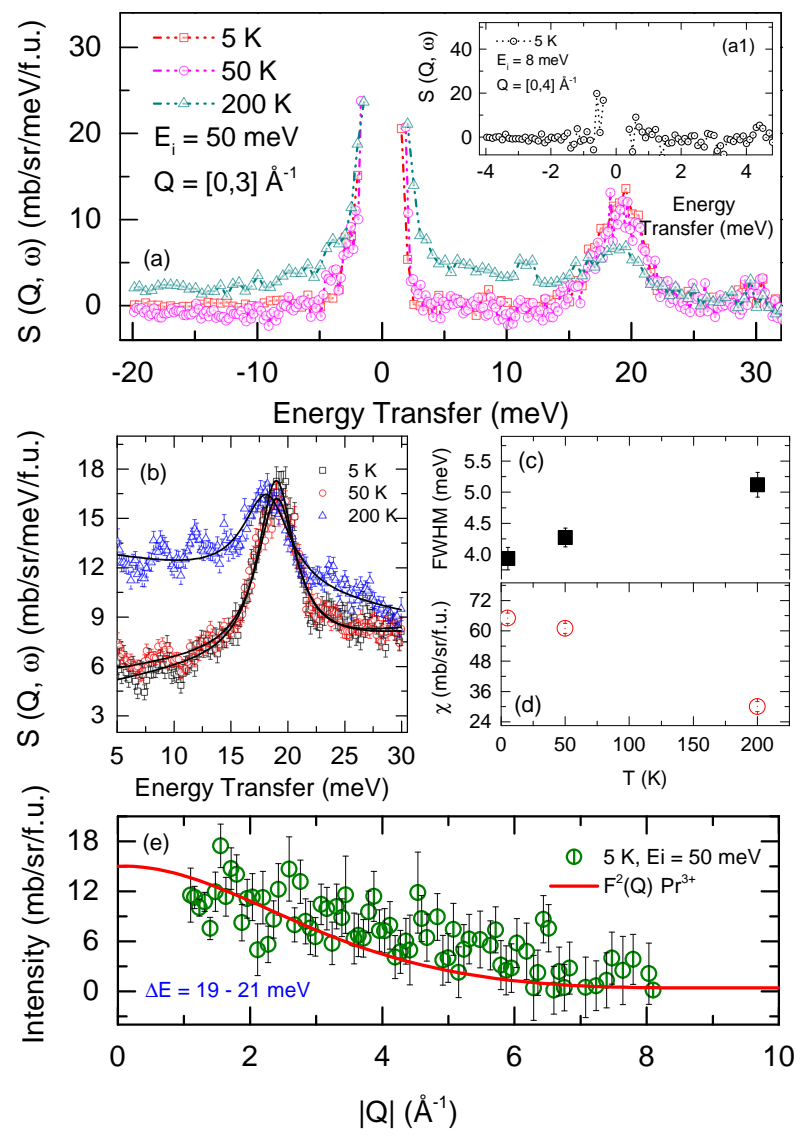

FIG. 6: (color online) (a) The dynamic structure factor $S(Q, \omega)$ of $\mathrm{PrFe}_{2} \mathrm{Al}_{8}$ at $5 \mathrm{~K}, 50 \mathrm{~K}$ and $200 \mathrm{~K}$ plotted as a function of energy transfer $\left(E_{i}=50 \mathrm{meV}\right)$. The inset (a1) shows the $S(Q, \omega)$ at $5 \mathrm{~K}$ at an incident energy $E_{i}=8 \mathrm{meV}$ where no excitation is observed. The curvefits (black lines), using a Bose-factor modified Lorentzian, to the $19 \mathrm{meV}$ excitation at $5 \mathrm{~K}, 50 \mathrm{~K}$ and $200 \mathrm{~K}$ are shown in (b). The temperature dependence of FWHM and the local susceptibility, estimated from the profile fits in (b) are shown in (c) and (d) respectively. In (e) the energy integrated intensity (19 to $21 \mathrm{meV}$ ) versus $|\mathrm{Q}|$ is plotted along with the magnetic form factor, $F^{2}(Q)$, for $\operatorname{Pr}^{3+}$. A peak observed near $12 \mathrm{meV}$ in the $200 \mathrm{~K}$ data is possibly extrinsic in origin.

suggested by the increasing FWHM and decreasing susceptibility. A peak is observed at $E \approx 12 \mathrm{meV}$ for the $200 \mathrm{~K}$ data (in the main panel of (a)) which, in fact, is narrower than the resolution and hence is judged as a spurious peak. The $|Q|$-dependence of integrated intensity at $5 \mathrm{~K}$, binned in the interval of $19-21 \mathrm{meV}$ is shown in the panel (e) plotted together with the square of the magnetic form factor, $F^{2}(Q)$ for $\mathrm{Pr}^{3+}$. The integrated intensity follows the $F^{2}(Q)$ for $\operatorname{Pr}^{3+}$ closely, suggesting that the inelastic excitation in $\mathrm{PrFe}_{2} \mathrm{Al}_{8}$ results from single ion effects of $\mathrm{Pr}^{3+}$. In order to estimate the magnetic moment from the inelastic signal at $19 \mathrm{meV}$, use was made of the second sum rule:

$$
\int_{-\omega}^{\omega} S(Q, \omega) / F^{2}(Q) d \omega=A \mu_{\mathrm{eff}}^{2}
$$

where $S(Q, \omega)$ is the dynamic structure factor, $F(Q)$ is the magnetic form factor, $A=48.6 \mathrm{mb} / \mathrm{sr} / \mu_{\mathrm{B}}^{2}$ is a constant[20] and $\mu_{\mathrm{eff}}$ is the effective magnetic moment. From the integrated intensity at the $19 \mathrm{meV}$ peak of $\mathrm{PrFe}_{2} \mathrm{Al}_{8}$ the effective moment value was estimated as $\mu_{\mathrm{eff}}=1.6(2) \mu_{\mathrm{B}}$ which is almost half of the spin-orbit coupled magnetic moment $\mu_{\mathrm{so}}^{\mathrm{Pr}}=3.58 \mu_{\mathrm{B}}$ of $\operatorname{Pr}^{3+}, J=4$. The low value of the moment associated with $19 \mathrm{meV}$ excitation along with the heat capacity results, which suggest a doublet CEF ground state, indicates that the missing Pr moment is associated with a narrow resolution limited quasi-elastic scattering from the ground state.

\section{DISCUSSION}

The specific heat of $\mathrm{PrFe}_{2} \mathrm{Al}_{8}$ showed a clear indication of a peak at $T_{N}^{\mathrm{Pr}} \approx 4 \mathrm{~K}$ where the Pr begins to order magnetically[14]. The magnetic contribution to the specific heat was obtained by subtracting the non-magnetic specific heat of $\mathrm{LaFe}_{2} \mathrm{Al}_{8}$. The estimate of crystal field levels were obtained by modeling the Schottky peak using a 9-level model for the crystal field levels. The first excited crystal field level was calculated at $\approx 10 \mathrm{~K}$ and from the behaviour of magnetic entropy as a function of temperature, a full occupation of the 9 levels was found to happen at $\approx 200 \mathrm{~K}$ where the entropy reaches $R \ln (9)$. From the inelastic neutron scattering data, a single excitation is discernible at $19 \mathrm{meV}$ which corresponds to $\approx 200 \mathrm{~K}$. The first excited level near $10 \mathrm{~K}(\approx 0.8 \mathrm{meV})$ is not observed in the inelastic data with either $E_{i}=8 \mathrm{meV}$ or $50 \mathrm{meV}$, which could be due to the zero matrix element between these wave functions - the intensity of CEF excitation vary as a square of this matrix element. However, since only one excitation is revealed in the inelastic scattering experiment, a full crystal field analysis to estimate all the 9 crystal field levels is not possible in the present case. Considering orthorhombic point symmetry $\left(C_{s}\right)$ of $\operatorname{Pr}$ ion in $\operatorname{PrFe}_{2} \mathrm{Al}_{8}$ there will be nine non-zero crystal field parameters in the Hamiltonian and hence a single observed CEF excitation is not sufficient to fit the CEF model. The temperature dependence of the inelastic excitation peak at $19 \mathrm{meV}$ shown in figure 6(a) indicates that the peak position does not shift with temperature, confirming the origin of the excitation to be crystal field effect. Moreover, the integrated intensity at $19 \mathrm{meV}$ as a function of $Q$ follows the square of form factor $F^{2}(Q)$ of $\operatorname{Pr}^{3+}$ confirming that the magnetism in $\operatorname{PrFe}_{2} \mathrm{Al}_{8}$ is purely from $\operatorname{Pr}^{3+}$. Though the previous magnetization study of $\mathrm{PrFe}_{2} \mathrm{Al}_{8}$ indicated short-range magnetic order due to $\mathrm{Fe}$, the neutron scattering results (figure 2) do not show any indication of this. The diffraction results only indicate that the long-range magnetic ordering in $\mathrm{PrFe}_{2} \mathrm{Al}_{8}$ at $4.5 \mathrm{~K}$ pertains only to the $\mathrm{Pr}$ sublattice where the moments order antiferromagnetically. The crystal field level scheme for $J=4, \mathrm{Pr}^{3+}$ in the local symmetry of $m$ predicts a singlet ground state. The $2 J+1=9$ fold multiplet of $\mathrm{Pr}^{3+}$ splits so that a crystal field singlet is always present for all type of crystallographic symmetry[21]. Pr-based intermetallics often display singlet ground states however, also have been found to lead to exotic behaviour such as the observation of heavy fermion superconductivity [22, 23]. Not 
only that, in certain Pr based systems like $\operatorname{PrAu}_{2} \mathrm{Si}_{2}$-related compounds, frustration effects on the magnetic ground state were argued to be introduced by crystal field effects[24]. In fact, a competition between the crystal field interaction and the magnetic exchange energy strengths were observed in these compounds, eventually, resulting in a novel spin glass ground state. Magnetic long range order also happens in several systems despite the crystal field level scheme warranting otherwise. PrIrSi $\mathrm{Pi}_{3}$ is one such system where Pr displays long range magnetic order[25]. However a large separation of about $90 \mathrm{~K}$ is observed between the first excited crystal field levels of PrIrSi $i_{3}$. Induced moment magnetic ordering in this compound would require very high internal exchange energy to achieve ordering. In some other Pr-based compounds like $\mathrm{PrRu}_{2} \mathrm{Si}_{2}$ and $\mathrm{PrNi}_{2} \mathrm{Si}_{2}$, relatively smaller crystal field separations are (of $2.25 \mathrm{meV}$ and $3.3 \mathrm{meV}$, respectively) [26, 27]. In the present case of $\mathrm{PrFe}_{2} \mathrm{Al}_{8}$ the knowledge of the first excited crystal field level first originated from a simulation of the Schottky specific heat, which predicts the first level at around $10 \mathrm{~K}$. An estimation of the exchange constant is in general possible through the relation, $\theta_{p}=\left[\frac{J_{\mathrm{ex}} J(J+1)}{3 k_{\mathrm{B}}}\right]$ where $\theta_{p}$ is the Curie-Weiss temperature and $J_{\text {ex }}$ is the exchange constant. However, in the case of $\mathrm{PrFe}_{2} \mathrm{Al}_{8}$ it was found that the inverse magnetic susceptibility did not follow a linear trend due to the presence of Fe short-range fluctuations and hence a mean-field estimate of $\theta_{p}$ was not possible. Comparing the ground state energy scale in $\mathrm{PrFe}_{2} \mathrm{Al}_{8}$ with other compounds like $\mathrm{PrIrSi}_{3}$ or with $\mathrm{PrRu}_{2} \mathrm{Si}_{2}$ and $\mathrm{PrNi}_{2} \mathrm{Si}_{2}$, it seems more plausible that an exchange-generated admixture of low lying crystal field energy levels is possible in the case of $\mathrm{PrFe}_{2} \mathrm{Al}_{8}$.

\section{CONCLUSIONS}

In conclusion, through neutron powder diffraction and inelastic scattering experiments we determined the magnetism of the quasi-skutterudite compound $\mathrm{PrFe}_{2} \mathrm{Al}_{8}$ Through the analysis of the neutron diffraction data, it is found that only Pr develops long-range magnetic order in this compound with a $T_{N}^{\mathrm{Pr}}=4.5 \mathrm{~K}$. The $\mathrm{Pr}^{3+}$ moments adopt antiferromagnetic arrangement with moments along the $c$-axis and a propagation vector $\mathbf{k}\left(\frac{\mathbf{1}}{\mathbf{2}} \mathbf{0} \frac{\mathbf{1}}{\mathbf{2}}\right)$. Only one magnetic excitation at $19 \mathrm{meV}$ is observed in the inelastic neutron scattering data of $\mathrm{PrFe}_{2} \mathrm{Al}_{8}$ corresponding to the crystal field excitation from $\mathrm{Pr}^{3+}$ levels. The presence of only one excitation detected in our experiments has rendered the estimation of the full crystal field level scheme of $\operatorname{Pr}^{3+}$ in $\operatorname{PrFe}_{2} \mathrm{Al}_{8}$ impossible as there are nine free crystal field parameters to be determined from the one excitation. However, from the $|Q|$-dependence of integrated magnetic intensity it is confirmed that the magnetism in $\mathrm{PrFe}_{2} \mathrm{Al}_{8}$ is solely governed by Pr while the Fe sublattice remains magnetically inactive down to $2 \mathrm{~K}$.

\section{Acknowledgements}

HSN acknowledges FRC/URC for a postdoctoral fellowship. AMS thanks the SA-NRF (93549) and the FRC/URC of UJ for financial assistance. DTA thanks CMPC-STFC grant number CMPC-09108 for the funding. We would like to thank A. Bhattacharyya for his help on MARI experiment.
[1] Sales B C, Mandrus D and Williams R K 1996 Science 272 1325

[2] Nolas G S, Morelli D T and Tritt T M 1999 Ann. Rev. Mater. Sci. 29 89-116

[3] Guo J Q, Geng H Y, Ochi T, Suzuki S, Kikuchi M, Yamaguchi $\mathrm{Y}$ and Ito S 2012 J. Elec. Mater. 41 1036-1042

[4] Frank F C and Kasper J S 1958 Acta Crystallogr. 11 184-190

[5] Tursina A I, Nesterenko S N, Murashova E V, Chernyshev I V, Nol H and Seropegin Y D 2005 Acta Crystallogr. E 61 i12-i14

[6] Adroja D T, Hillier A D, Muro Y, Takabatake T, Strydom A M, Bhattacharyya A, Daoud-Aladin A and Taylor J W 2013 Physica Scripta $\mathbf{8 8} 068505$

[7] Takeda N and Ishikawa M 2000 J. Phys. Soc. Jpn. 69 868-873

[8] Adroja D T, Hillier A D, Park J G, Goremychkin E A, McEwen K A, Takeda N, Osborn R, Rainford B D and Ibberson R M 2005 Physical Review B 72184503

[9] Ito T U, Wataru H, Kazuhiko N, Hubertus L, Christopher B, Akito S and Satoru N 2011 J. Phys. Soc. Jpn. 80113703

[10] Masahito S, Kazuhiro K, Shuhei T, Eiichi M, Hitoshi S, Matsuda T D and Yoshinori H 2012 J. Phys. Soc. Jpn. 81 SB011

[11] Ślebarski A, Goraus J, Witas P, Kalinowski L and Fijałkowski M 2015 Phys. Rev. B 91035101

[12] Khuntia P, Peratheepan P, Strydom A M, Utsumi Y, Ko K T, Tsuei K D, Tjeng L H, Steglich F and Baenitz M 2014 Phys. Rev. Lett. 113216403

[13] Snyman J L and Strydom A M 2012 J. Appl. Phys. 111 07A943
[14] Nair H S, Ghosh S K, Rameshkumar K and Strydom A M 2016 J. Phys. Chem. Solids 91 69-75

[15] Azuah R T, Kneller L R, Qiu Y, Tregenna-Piggott P L W, Brown C M, Copley J R D and Dimeo R M 2009 J. Res. Nat. Inst. Standards and Tech. 114341

[16] Rietveld H M 1969 J. Appl. Crystall. 2 65-71

[17] Rodriguez-Carvajal J 2010 LLB, CEA-CNRS, France [http://www. ill. eu/sites/fullprof/]

[18] Wills A S 2000 Physica B 276 680-681

[19] Tougait O, Kaczorowski D and Noël H 2005 J. Solid State Chem. 178 3639-3647

[20] Holland-Moritz E, Wohlleben D and Loewenhaupt M 1982 Phys. Rev. B 257482

[21] Walter U 1984 J. Phys. Chem. Solids 45 401-408

[22] Bauer E D, Frederick N A, Ho P C, Zapf V S and Maple M B 2002 Phys. Rev. B 65100506

[23] E A Goremychkin, Osborn R, Bauer E D, Maple M B, Frederick N A, Yuhasz W M, Woodward F M and Lynn J W 2004 Phys. Rev. Lett. 93157003

[24] Goremychkin E A, Osborn R, Rainford B D, Macaluso R T, Adroja D T and Koza M 2008 Nat. Phys. 4 766-770

[25] Anand V K, Adroja D T, Bhattacharyya A, Hillier A D, Taylor J W and Strydom A M 2014 J. Phys.: Condens. Matter 26 306001

[26] A M Mulders, Yaouanc A, Dalmas de Rotier P, Gubbens P C M, Moolenaar A A, Fk B, Ressouche E, Proke K, Menovsky A A 
and Buschow K H J 1997 Phys. Rev. B 56 8752-8759

[27] Blanco J A, Gignoux D and Schmitt D 1992 Phys. Rev. B 45 